\title{
Effects of synbiotic supplementation on energy and macronutrients homeostasis and muscle wasting of critical care patients: study protocol and a review of previous studies
}

\author{
Najmeh Seifi ${ }^{1}$, Mohammad Safarian ${ }^{2+}$, Mohsen Nematy ${ }^{2}$, Reza Rezvani ${ }^{1}$, Majid Khadem-Rezaian ${ }^{3}$ and
} Alireza Sedaghat ${ }^{4^{*}+}$

\begin{abstract}
Background: An extreme and persistent dysbiosis occurs among critically ill patients, regardless of the heterogeneity of disease. Dysbiosis in critically ill patients may make them prone to hospital-acquired infections, sepsis, multi-organ failure (MOF), energy homeostasis disturbance, muscle wasting, and cachexia. Modulation of gut microbiota through synbiotics can be considered as a potential treatment for muscle wasting and macronutrient homeostasis disturbances.

Methods: This is a prospective, single-center, double-blind, parallel randomized controlled trial with the aim to evaluate the effects of synbiotic supplementation on energy and macronutrient homeostasis and muscle wasting in critically ill patients. A total of 40 hemodynamically stable, adult, critically ill patients who receive enteral nutrition via a nasogasteric tube (NGT) in the 24-48 h after admission to critical care will be included in this study. Eligible patients will be randomly assigned to receive Lactocare (ZistTakhmir) capsules $500 \mathrm{mg}$ every $12 \mathrm{~h}$ or a placebo capsule, which contains only the sterile maize starch and is similar to synbiotic capsules for 14 days. The synbiotic and placebo capsules will be given through the nasogastric tube, separately from gavage, after feeding.

Discussion: Gut microbiota modulation through synbiotics is proposed to improve clinical prognosis and reduce infectious complications, ventilator dependency, and length of ICU stay by improving energy and macronutrient homeostasis and reducing muscle protein catabolism.
\end{abstract}

Trial registration: Iranian Registry of Clinical Trials, IRCT20190227042857N1. Registered on 17 March 2019.

Keywords: Synbiotics, Homeostasis, Muscle wasting, Critical care, Gut microbiota

\section{Background}

\section{Gut microbiota, dysbiosis and critical illness}

The gut microbiota refers to the commensal microorganisms that reside in our gastrointestinal tract (GIT) with a symbiotic relationship [1]. Gut microbiota has a significant role in host metabolism and homeostasis [2, 3]. A disturbance in

\footnotetext{
* Correspondence: Sedaghatar@mums.ac.ir

${ }^{\dagger}$ Mohammad Safarian and Alireza Sedaghat contributed equally to this work.

${ }^{4}$ Department of Anesthesiology, Mashhad University of Medical Sciences,

Mashhad, Iran

Full list of author information is available at the end of the article
}

this microbial community, which leads to an unhealthy state, is called dysbiosis [4]. Over the past decade, emerging evidence has demonstrated the role of intestinal dysbiosis in the pathogenesis of various conditions, such as infectious, immune, and metabolic diseases [5], while it has not been studied extensively in critical illness. An extreme and persistent dysbiosis occurs among critically ill patients, regardless of the heterogeneity of disease. The extreme dysbiosis in patients under critical care is due to the stress of critical illness, multiple antibiotics and additional pharmacological interventions, and highly processed enteral/parenteral 
nutrition [6, 7]. Dysbiosis in critically ill patients may make them prone to hospital-acquired infections, sepsis, multi-organ failure (MOF), energy homeostasis disturbance, muscle wasting, and cachexia $[6,8,9]$.

\section{Dysbiosis and energy homeostasis in critical illness}

The majority of patients in the intensive care unit (ICU) have had a severe illness, trauma, or major surgery, and accordingly they are unable to manage their nutritional demands. Although nutritional support is a daily practice in the ICU, many patients still suffer from malnutrition due to lack of intake or uptake of nutrients [10]. The prevalence of malnutrition in the ICU within developed and developing countries is reported as $50.8 \%$ and $78.1 \%$, respectively [11]. Malnutrition is independently associated with longer ICU stay, more ICU readmissions, and a higher incidence of infections and risk of mortality [11]. A greater degree of malnutrition is also associated with a higher risk of 28-day mortality [12]. Malnutrition further tends toward acute or chronic loss of muscle bulk and function [13]. The gut microbiota and their derived metabolites play an essential role in the absorption, storage, and consumption of energy derived from the diet $[14,15]$. Previous studies suggest that modulating gut microbiota by novel therapeutics, such as prebiotics, probiotics, or synbiotics, can have an effect on gastrointestinal tolerance and complications of enteral nutrition, which eventually lead to the regulation of energy intake. Recently, Tuncay et al. showed that enteric formula with prebiotic content in patients under neurocritical care was associated with a significant increase in total feed volume and energy intake and a non-significant tendency to achieve a target dose of nutrition more frequently and earlier [16]. Malik et al. also found that in patients in the ICU, receiving enteral formula supplemented with probiotics led to a faster return of gut function (tolerated feeding of $80 \%$ of their estimated required calories for $48 \mathrm{~h}$ consecutively) [17]. However, Sanaie et al. demonstrated that daily energy and protein intake in patients receiving probiotic supplements on the ICU were not significantly different from the group receiving placebo [18]. In the critical care setting, diarrhea is the most obvious complication of enteral nutrition (EN), which is associated with the inadequacy of energy and macronutrient intake [16]. Previous systematic reviews have confirmed the significant benefit of probiotics in the reduction of diarrhea in hospital patients overall. But a recent meta-analysis focused on patients in the ICU found no benefit of probiotics in preventing or treating diarrhea [19]. Besides, in the dysbiosis state of critical illness, microbial products that reach distant organs like brain, adipose tissue, and liver, favor the development of immune-mediated diseases and metabolic alterations [20]. Gut microbiota metabolites like short-chain fatty acids (SCFAs) can also influence the immune system and host metabolism, which regulates energy homeostasis [20]. Thus, gut microbiota modulation may be beneficial in the regulation of immune and metabolic responses and energy homeostasis.

\section{Dysbiosis and muscle wasting in critical illness}

Muscle wasting, characterized by loss of muscle mass and strength, is associated with negative health outcomes such as functional disability, greater risk of infections, delayed recovery, poor life quality, and mortality [21]. The gut microbiota have been reported to influence muscle metabolism. The molecular mechanisms of this gut-muscle axis remain to be identified. Gut microbiota influence amino acid bioavailability and are sources of different metabolites, such as conjugated linoleic acid, acetate, and bile acids, which modulate muscle metabolism [8]. Various pathogen-associated molecular patterns (PAMPs) activate the transcription factor nuclear factor kappa light chain enhancer of activated B cells (NF-kB) through toll-like receptors (TLRs) and modulate the production of proinflammatory cytokines, which can induce muscle atrophy [8] (Fig. 1).

Modulation of gut microbiota through prebiotics, probiotics, or synbiotics can be considered as a potential treatment for muscle wasting and cachexia. In mouse models of leukemia, restoring Lactobacillus species by oral supplementation with Lactobacillus reuteri 100-23 and Lactobacillus gasseri 311,476 reduces inflammatory cytokines and expression of muscle atrophy makers [22]. In another study, Bindels et al. showed that prebiotic supplementation in leukemic mice could contribute to delaying anorexia and fat mass sparing by inducing a metabolic shift in adipose tissue [23]. In the mouse models of cancer cachexia, administration of a synbiotic supplement including inulin-type fructans and live $L$. reuteri 100-23 was associated with restoration of the gut barrier and immune function, thus reducing cachexia. It also prolonged survival [24]. Varian et al. also showed that probiotic administration in leukemic mice could inhibit cachexia by reducing systemic inflammation [25].

\section{Study rationale}

Considering the extreme dysbiosis in critically ill patients and related energy and macronutrients homeostasis disturbance and muscle wasting, prompted us to evaluate the effect of synbiotic supplementation on the elimination of this condition. To our knowledge, this is the first study to investiage the effect of synbiotic supplementation on muscle wasting in critically ill patients.

\section{Study objectives \\ Primary objective}

The primary objective is to evaluate the effects of synbiotic supplementation on energy and macronutrient homeostasis and muscle wasting in patients under critical care. 


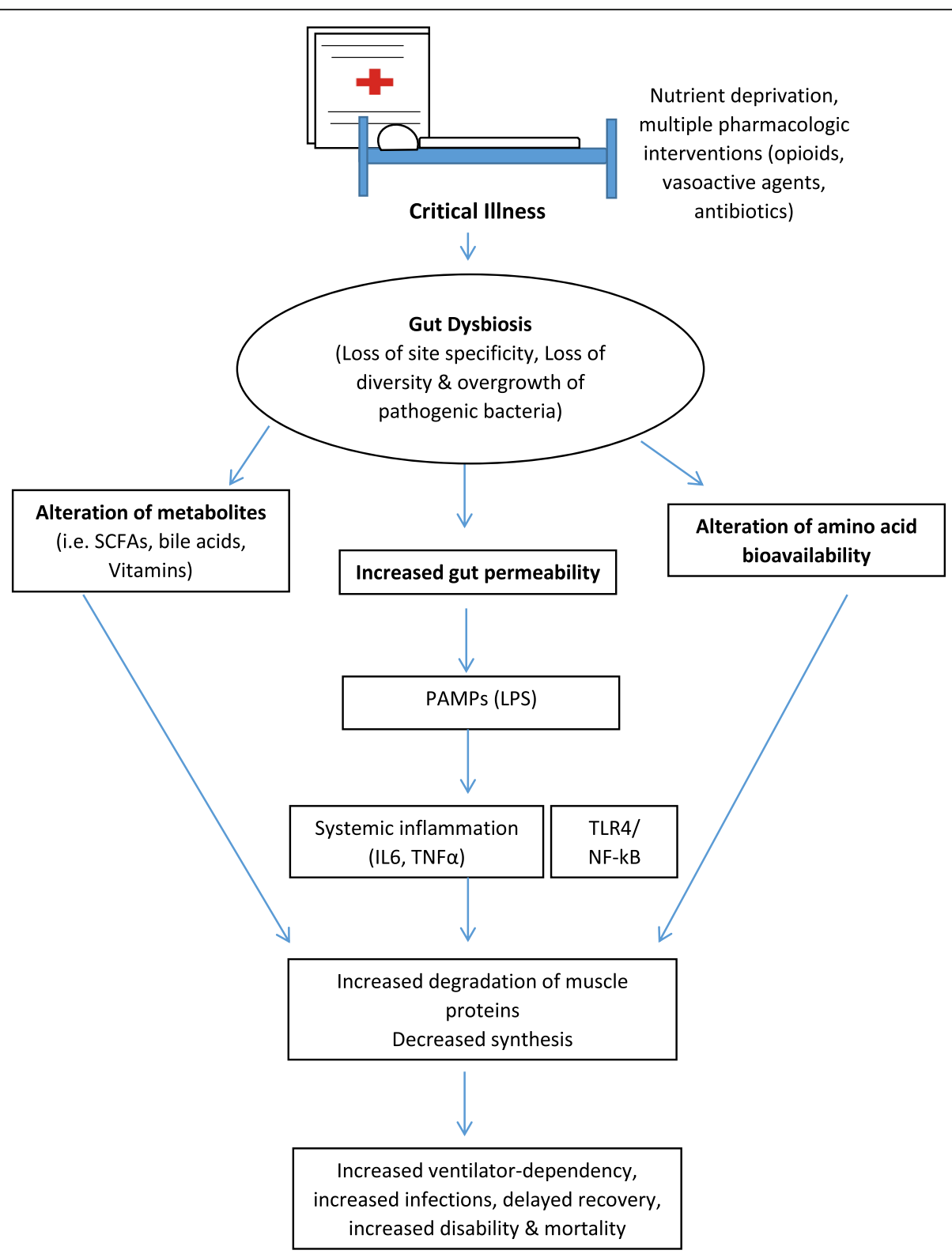

Fig. 1 Dysbiosis and muscle wasting in critical illness. Abbreviations: EN, enteral nutrition; IL6, interleukin 6; LPS, lipopolysaccharides; NF-kB, nuclear factor kappa light chain enhancer of activated B cells; PAMPs, pathogen-associated molecular patterns; SCFAs, short-chain fatty acids; TLR4, toll-like receptor 4; TNFa, tumor necrosis factor alpha

\section{Secondary objectives}

The secondary objective is to evaluate the effects of synbiotic supplementation on infectious complications and length of hospital and ICU stay in patients under critical care.

\section{Study design}

This is a prospective, single-center, double-blind, parallel randomized controlled trial that will be conducted in Edalatian Medical ICU, Emam Reza Hospital, Mashhad, Iran. The study protocol was written following the Standard Protocol
Items: Recommendation for Interventional Trials (SPIRIT) Checklist (Additional file 1).

\section{Selection and enrollment of participants} Inclusion criteria

Participants must meet all the inclusion criteria to participate in this study: adults aged 18-65 years; admitted to the ICU; hemodynamically stable within $24-48 \mathrm{~h}$ after admission; requiring exclusive enteral nutrition (EN) via a nasogastric tube (NGT); not taking any kind of microbial cell preparations (prebiotics probiotics, synbiotics); estimated length of ICU stay more than 14 days; and provision of written consent. 


\section{Exclusion criteria}

All candidates meeting any of the exclusion criteria at baseline will be excluded from study participation: pregnancy or lactation; any contraindication to EN; any contraindication to insertion of the NGT; receiving immunosuppressive treatment, radiotherapy, or chemotherapy; hematologic disease; acquired immune deficiency syndrome (AIDS); transplant recipient; known allergy to microbial cell preparations; cancer or autoimmune diseases; artificial heart valve or congenital heart valve disease; estimated length of ICU stay less than 4 days; gastric disease; or gastrointestinal tract (GI) tract surgery.

\section{Study enrollment procedure}

Before the screening procedure, informed consent will be obtained from every participant who meets the inclusion criteria. First, we will describe the purpose of the study, the procedures involved, the length of time the subject is suspected to participate, any possible disadvantages or discomforts, the benefits of the study to society and individuals, and the person to contact for answers to further questions. We will also emphasize that participation is voluntary, and refusal or withdrawal will not cause any loss of benefits that they are entitled to receive. Then the participants or their legal guardian will read and sign two copies of the written form. If, the informed consent was obtained from the patient's guardian because of the patient's lack of competence to consent and then later in the study the patient subsequently became competent as required, consent will be regained.

\section{Random allocation and blinding}

After providing their written consent, patients are randomly allocated in a 1:1 ratio to the intervention or control group (A or B). Patients will be randomized through a stratified sequential randomization plan generated online. Randomization will be stratified by disease severity (Acute Physiology and Chronic Health Evaluation (APACHE) II, ${ }^{1} 0-35$ and 35-70). For allocation concealment, we will use sealed opaque envelopes; inside each there is a carbon paper and the A or B card. To avoid probable selection bias, we will write the patient's name on the envelope before opening it. All patients, researchers, and medical staff will be blinded to the allocation to either synbiotic or placebo capsules. An available third party, the secretary of the ICU, will be aware of whether A or $B$ is allocated the synbiotic supplement. In the case of any complication associated with the intervention allocated, the medical staff will refer to the secretary for details.

\section{Study interventions}

\section{Interventions, administration, and duration}

All eligible patients will receive standard hospital gavage as EN through the NGT in the 24-48 $\mathrm{h}$ after admission to the

\footnotetext{
${ }^{1}$ Acute Physiology and Chronic Health Evaluation II
}

ICU. According to the recent European Society of Parenteral and Enteral Nutrition (ESPEN) guideline on clinical nutrition in the ICU [26], continuous rather than bolus EN is preferred because it causes less diarrhea, but there is no difference in other outcomes. Another systematic review showed that bolus feeding is associated with lower aspiration rate and better calorie attainment [27]. It also provides a greater stimulus for protein synthesis [28]. Considering these data and the availability of bolus EN in our hospitals we applied this method. In the absence of an indirect calorimeter, the simple weight-based equation of $20-25 \mathrm{Kcal} / \mathrm{kg} /$ day in the acute flow phase and $25-30 \mathrm{Kcal} / \mathrm{kg} / \mathrm{day}$ in the anabolic flow phase is preferred for measurement of calorie requirements. For overweight and obese patients, ideal body weight: $0.9 \times$ height $(\mathrm{cm})-100$ (male) (or - 106 (female) is suggested as a reference weight [26]. To avoid overfeeding, the EN target will be prescribed within 3 days in patients with high nutritional risk and within 7 days in patients with low nutritional risk according to the modified Nutrition Risk in Critically Ill (NUTRIC) $^{2}$ score. The flow charts in Figs. 2 and 3 will be used for initiation and continuation of EN.

In the intervention group, patients will receive Lactocare (ZistTakhmir) capsules $500 \mathrm{mg}$ every $12 \mathrm{~h}$ for 14 days. Each capsule contains Lactobacillus casei $1.5 \times 10^{9}$ colony-forming units (CFU), Lactobacillus acidophilus $1.5 \times 10^{10} \mathrm{CFU}$, Lactobacillus rhamnosus $3.5 \times 10^{9} \mathrm{CFU}$, Lactobacillus bulgaricus $2.5 \times$ $10^{8} \mathrm{CFU}$, Bifidobacterium breve $1 \times 10^{10} \mathrm{CFU}$, Bifidobacterium longum $5 \times 10^{8} \mathrm{CFU}$, Streptococcus thermophilus $1.5 \times 10^{8}$ $\mathrm{CFU}$, and fructooligosaccharides (FOS). The probiotics capsule will be given through the NGT, separately from gavage, after feeding. Patients in the control group will receive a placebo capsule, which contains only sterile maize starch and is similar to probiotic capsules. The liquid preparations ready for gavage through the NGT are also similar in color and odor.

\section{Handling of the study intervention}

The pharmaceutical company will provide synbiotic and placebo capsules in distinct boxes identified as A or B. Synbiotic capsules can be stored at room temperature for 2-3 weeks but the best condition for keeping this product is in the refrigerator at $2-8{ }^{\circ} \mathrm{C}$. Unused study products will be returned to the company supplying them.

\section{Concomitant interventions \\ Concomitant interventions will be:}

\section{Antibiotics}

- It is common that patients under critical care receive at least one antibiotic during their ICU stay.

${ }^{2}$ Nutrition Risk In Critically Ill 


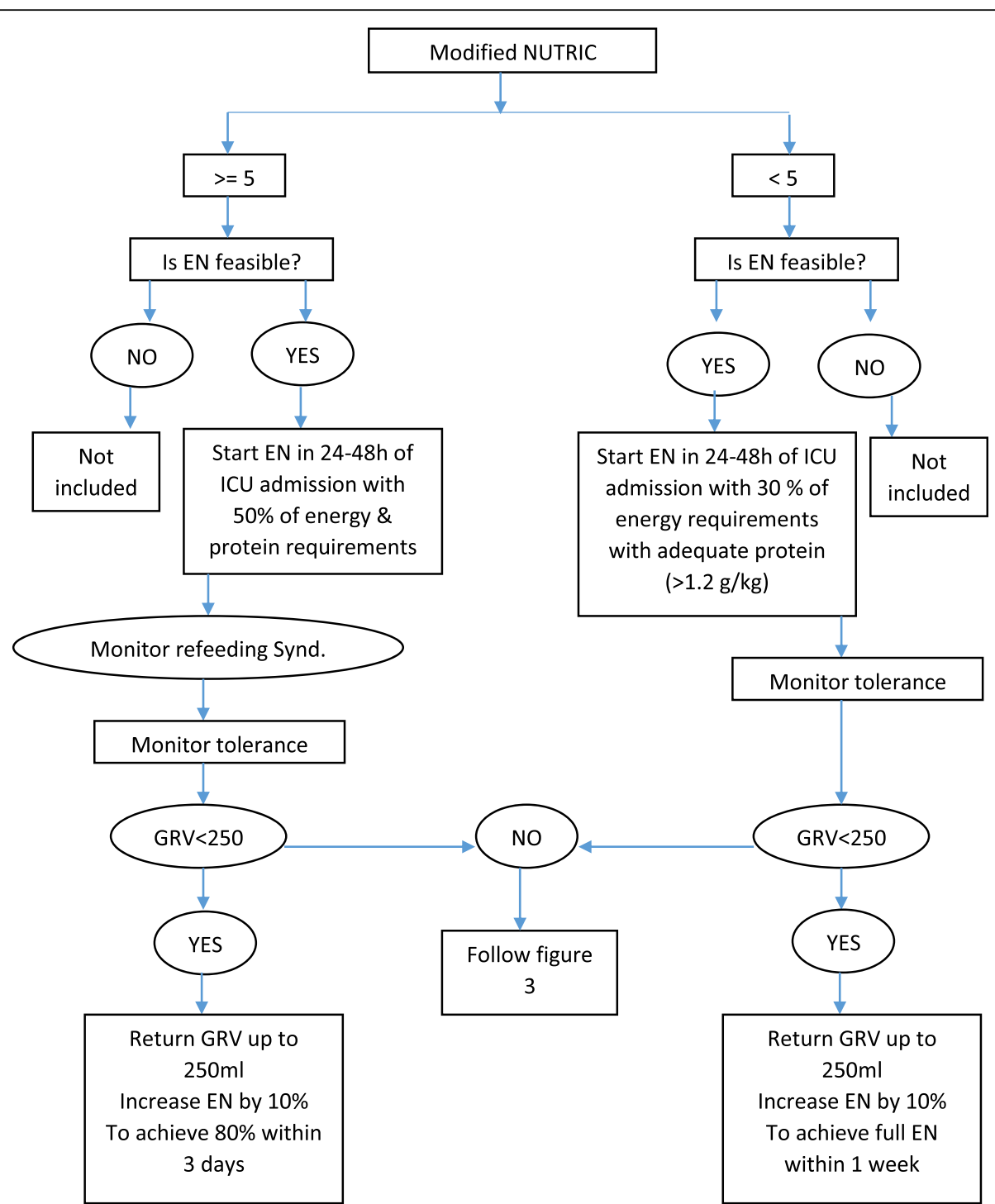

Fig. 2 Flow chart for EN initiation and goal achievement. Abbreviations: EN, enteral nutrition; GRV, gastric residual volume; ICU, intensive care unit; ml, milliliter; NUTRIC, Nutrition Risk in Critically III

On the other hand, it is believed that antibiotics have bacteriostatic or bactericidal effects on both pathogenic and non-pathogenic bacteria. So, it is recommended that probiotic and antibiotic administration be separated by at least $2 \mathrm{~h}$ hours [29]. care. Opioids are believed to suppress the immune system and delay GI peristalsis [30]. Delayed peristalsis can increase bacterial translocation out of the GI tract [31].

2. Opioids

3. H2 receptor blockers

- Considering their analgesic and sedative properties, opioids are widely used in patients under critical

- Prevention of GI tract (GIT) stress ulcers, through $\mathrm{H} 2$ receptor blockers or proton-pump inhibitors, is common in critical care practice. Increase in GI 


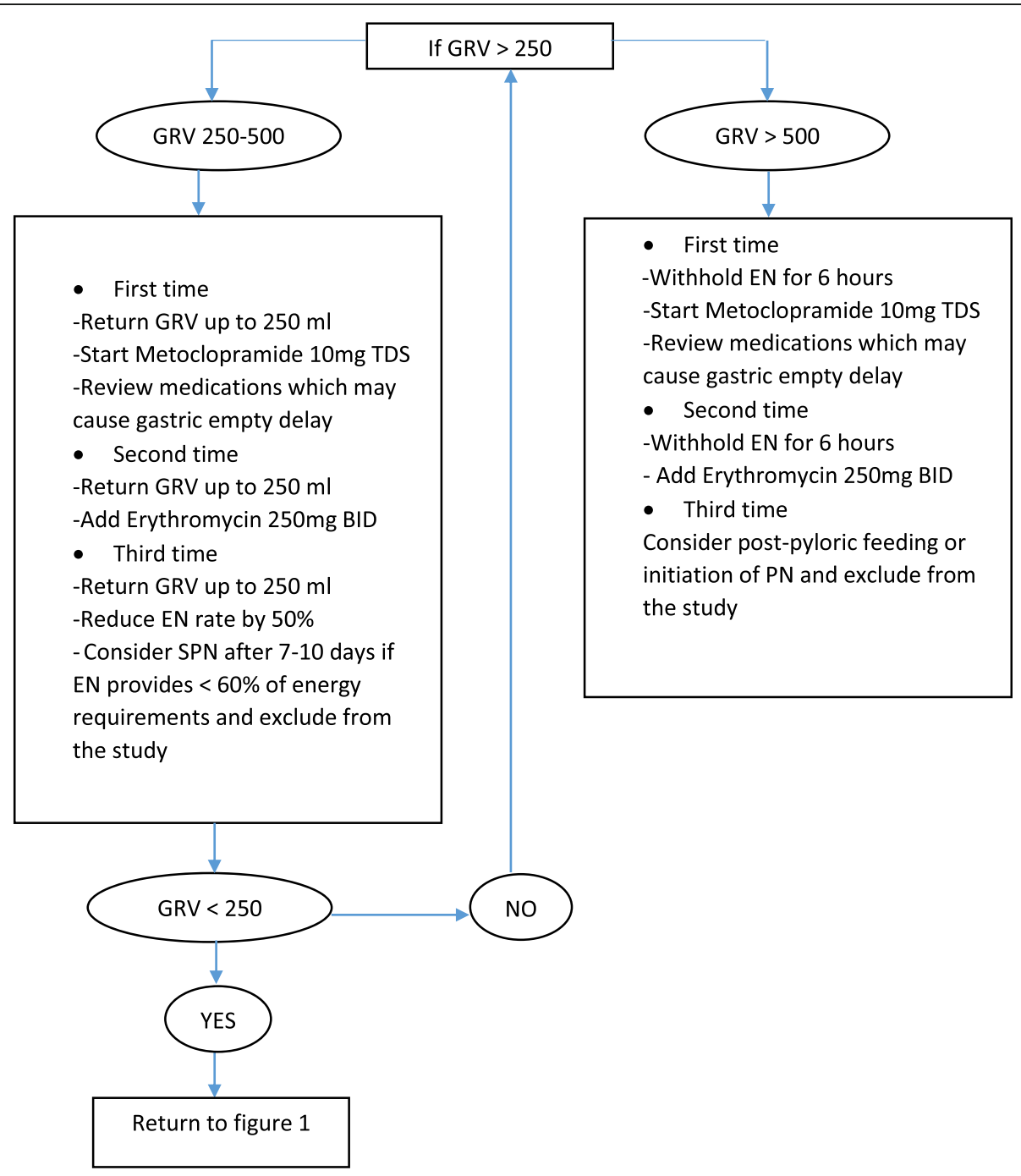

Fig. 3 Flow chart of GRV management. Abbreviations: EN, enteral nutrition; GRV, gastric residual volume; ICU, intensive care unit; ml, milliliter; NUTRIC, Nutrition Risk in Critically III; SPN, supplementary parenteral nutrition

acidity can cause overgrowth of some pathogenic bacteria [32].

4. Catecholamines

- It is believed that the elevated level of catecholamines in patients under critical care, as prescribed exogenously beside endogenous production, can impair the immune system [33].

These drugs are routinely administered in critical care practice, so we will record and consider them as conflicting factors.

\section{Adherence assessment}

As the researcher will administer the capsules to the patients through the NGT, adherence assessment is not required.

\section{Study procedures}

Schedule of evaluations

The schedule of evaluations is shown in Table 1.

\section{Description of evaluations}

As shown in Fig. 2, calorie achievement goals are set according to the patients' modified NUTRIC score. In everyday visits, we will evaluate GI signs and symptoms (e.g. vomiting, diarrhea, abdominal distention) and gastric residual volume (GRV). If there is no sign or symptom of intolerance and GRV is less than $250 \mathrm{ml}$, EN will be increased by $10 \%$. Otherwise, we will approach as 
Table 1 Schedule of enrollment, intervention, and assessments in accordance with the Standard Protocol Items: Recommendations for Interventional Trials (SPIRIT) guidelines

\begin{tabular}{|c|c|c|c|c|c|c|c|c|c|c|c|c|c|c|c|c|c|}
\hline \multicolumn{18}{|l|}{ Study period } \\
\hline \multirow[b]{2}{*}{ Time point } & \multirow{2}{*}{$\begin{array}{l}\text { Enrollment } \\
\text { Pre- allocation }\end{array}$} & \multirow{2}{*}{$\begin{array}{l}\text { Allocation } \\
\text { Pre- intervention }\end{array}$} & \multicolumn{14}{|c|}{ Post-allocation } & \multirow{2}{*}{$\begin{array}{l}\text { Close- out } \\
15\end{array}$} \\
\hline & & & 1 & 2 & 3 & 4 & 5 & 6 & 7 & 8 & 9 & 10 & 11 & 12 & 13 & 14 & \\
\hline \multicolumn{18}{|l|}{ Enrollment } \\
\hline Informed consent form & $\times$ & & & & & & & & & & & & & & & & \\
\hline Eligibility & $x$ & & & & & & & & & & & & & & & & \\
\hline Demographics & $\times$ & & & & & & & & & & & & & & & & \\
\hline Intervention & & & $\times$ & $\times$ & $\times$ & $x$ & $\times$ & $\times$ & $\times$ & $x$ & $\times$ & $x$ & $\times$ & $\times$ & $\times$ & $\times$ & \\
\hline \multicolumn{18}{|l|}{ Assessments } \\
\hline APACHEII, Modified NUTRIC, SOFA & & $\times$ & & & & & & & & $\times$ & & & & & & & $\times$ \\
\hline GCS and vital signs & & $\times$ & $\times$ & $\times$ & $\times$ & $\times$ & $\times$ & $\times$ & $x$ & $x$ & $\times$ & $\times$ & $\times$ & $\times$ & $\times$ & $\times$ & \\
\hline Energy homeostasis & & $\times$ & $\times$ & $x$ & $\times$ & $\times$ & $\times$ & $\times$ & $x$ & $\times$ & $\times$ & $\times$ & $\times$ & $\times$ & $\times$ & $\times$ & $\times$ \\
\hline Abdominal examination & & $\times$ & $\times$ & $\times$ & $\times$ & $\times$ & $\times$ & $\times$ & $\times$ & $x$ & $\times$ & $x$ & $x$ & $\times$ & $x$ & $\times$ & \\
\hline Fluid balance examination & & $\times$ & $\times$ & $x$ & $\times$ & $\times$ & $x$ & $x$ & $\times$ & $x$ & $\times$ & $x$ & $\times$ & $\times$ & $x$ & $\times$ & \\
\hline GRV & & & & & & & & & & & & & & & & & According to Fig. 3. \\
\hline Mid-arm circumference & & $\times$ & $\times$ & & & $\times$ & & & $x$ & & & $\times$ & & & $x$ & & $x$ \\
\hline Infectious events & & $\times$ & $\times$ & $\times$ & $\times$ & $\times$ & $\times$ & $\times$ & $\times$ & $x$ & $\times$ & $\times$ & $\times$ & $\times$ & $\times$ & $\times$ & $\times$ \\
\hline Pressure ulcer & & $\times$ & $\times$ & $x$ & $\times$ & $x$ & $\times$ & $\times$ & $\times$ & $\times$ & $\times$ & $\times$ & $x$ & $\times$ & $\times$ & $x$ & $\times$ \\
\hline $\mathrm{Mg}, \mathrm{P}, \mathrm{Na}, \mathrm{K}, \mathrm{Cl}$ & & $\times$ & $\times$ & $\times$ & $\times$ & $\times$ & $\times$ & $\times$ & $\times$ & $\times$ & $\times$ & $\times$ & $x$ & $\times$ & $x$ & $\times$ & \\
\hline Glucose & & $\times$ & $\times$ & $\times$ & $\times$ & $\times$ & $\times$ & $\times$ & $\times$ & $\times$ & $\times$ & $\times$ & $\times$ & $\times$ & $\times$ & $\times$ & $\times$ \\
\hline Insulin & & $\times$ & & & & & & & & & & & & & & & $\times$ \\
\hline AST, ALT,TG, Urea & & $\times$ & & & & $\times$ & & & $\times$ & & & $\times$ & & & $\times$ & & \\
\hline PreAlb & & $\times$ & & & & & & & & $\times$ & & & & & & & $\times$ \\
\hline Outcomes & & $\times$ & & & & & & & & & & & & & & & $\times$ \\
\hline Concomitant medication & & $\times$ & $\times$ & $\times$ & $\times$ & $\times$ & $\times$ & $\times$ & $\times$ & $\times$ & $\times$ & $\times$ & $\times$ & $\times$ & $\times$ & $\times$ & \\
\hline Adverse events & & $\times$ & $\times$ & $\times$ & $\times$ & $\times$ & $\times$ & $\times$ & $\times$ & $\times$ & $x$ & $\times$ & $\times$ & $\times$ & $\times$ & $\times$ & \\
\hline Mortality rate & & & & & & & & & & & & & & & & & After 28 days \\
\hline
\end{tabular}

Abbreviations: ALT alanine aminotransferase, AST aspartate aminotransferase, APACHE II Acute Physiology and Chronic Health Evaluation II, Cl chloride, Cr creatinine, GCS Glasgow coma scale, GRV gastric residual volume, $K$ potassium, Mg magnesium, Na sodium, NUTRIC Nutrition Risk in Critically III, $P$ phosphorus, PreAlb pre-albumin, SOFA sequential organ failure assessment, TG triglyceride

shown in Fig. 3. Energy homeostasis (calorie intakeestimated calorie requirement) will be recorded each day. Mid-arm circumference, which is an available anthropometric measurement tool, will be evaluated twice a week. As all patients receiving EN should be monitored for some clinical and laboratory variables, we set our monitoring approach as reported by Berger MM, et al. [34], Monitoring nutrition in the ICU, Clinical Nutrition (2018). Concomitant medications, pressure ulcers, infectious complications, and other adverse events will be recorded every day. The APACHE II and Sequential Organ Failure Assessment (SOFA) will be scored on days 0,8 , and 15 . Before and after the intervention, fasting blood and 24-h urine samples will be obtained. Urine urea nitrogen, 3-Methyl histidine, and creatinine will be measured in urine samples. Fasting blood glucose, insulin, C-reactive protein (CRP) and endotoxins will be measured in fasting blood samples.

\section{Safety assessment}

Despite the ample evidence supporting the safety of probiotics in critically ill patients, there are case reports of risks and suggested theoretical risks related to probiotic administration. The most important is the risk of bacteremia and fungemia in high-risk populations, which may be associated with improper use and unintended contamination of central-line catheters [33]. To avoid risk of bacteremia we will not include a high-risk population, such as patients who have recently had major surgery, or patients with short bowel syndrome, 
heart valve disease, artificial heart valve, or patients who are immunocompromised. We will also pay careful attention to the proper administration and handwashing protocols. Gene transfer and over-stimulation of the immune system are other suggested theoretical risks, on which there is not yet any evidence in humans [33].

\section{Intervention discontinuation}

If intervention-related side effects exceed the level reported by previous studies, we will stop the intervention and present the results to the Ethics Committee of Mashhad University of Medical Sciences (MUMS) for further decision making. If adverse events are caused by the study intervention, the researcher and medical team will provide timely and proper treatment to participants.

\section{Statistical considerations General design issues}

Data will be analyzed using the intention-to-treat approach.

\section{Sample size and randomization}

We did not find any similar study that has evaluated our primary objectives. So, we considered one of the main secondary objectives to estimate the required sample size. Mahmoodpoor and co-workers [35] reported the ICU stay in two study groups as $18.6 \pm 8$ and $11.6 \pm 6.3$ days. Considering alpha error of 0.05 and power of $80 \%$, the required sample size allowing for $10 \%$ dropout is 20 patients in each group.

\section{Outcomes}

\section{Primary outcomes}

The primary outcomes are

1. Energy homeostasis (calorie intake-estimated calorie requirement)

2. Protein catabolism (nitrogen balance)

- Nitrogen balance is a measure of the net change in total body protein. It is the difference between nitrogen eliminated from the body and nitrogen ingested in the diet. A positive or neutral nitrogen balance shows that protein stores are increased or maintained, while a negative nitrogen balance indicates protein mass is decreasing. The practical method for estimating nitrogen balance supposes that total nitrogen loss is equal to urinary urea nitrogen excretion plus $4 \mathrm{~g} /$ day additional loss from non-urinary urea nitrogen, gastrointestinal, and insensible losses [36, 37]. To measure the nitrogen balance, during the 24-h urine collection, the total intake of protein will be recorded to calculate nitrogen intake: 24-h urine samples will be immediately delivered to the laboratory to measure urea nitrogen.

3. Muscle protein degradation (3-methyl histidine (3MH) in 24-h urine)

- $3 \mathrm{MH}$ is exclusively found in muscle proteins, and after protein degradation, it is rapidly excreted in the urine without further reutilization or metabolization. So, measuring urinary $3 \mathrm{MH}$, after at least 1 day of a meat-free diet, can be used as a biomarker of muscle protein breakdown [38, 39]. After a 1-day meat-free diet, 24-h urine will be collected. Urine samples will be centrifuged for $20 \mathrm{~min}$ at $1000 \times \mathrm{g}$. The supernatant will be collected and stored at $70{ }^{\circ} \mathrm{C}$ for a maximum of 2 months. The ELISA method will be used for $3 \mathrm{MH}$ detection.

4. Muscle protein turnover $(3 \mathrm{MH} /$ creatinine ratio in 24-h urine)

- Since 24-h urinary creatinine estimates the total pool of muscle proteins, muscle protein turnover can be calculated from the $3 \mathrm{MH} /$ creatinine excretion ratio [38]. A 24-h urine sample will be delivered to the laboratory to immediately measure creatinine by the enzymatic method.

5. Lipolysis (free glycerol in serum)

- Free glycerol is an important index of lipid metabolism. When the body uses stored fat as the energy supply, glycerol and fatty acids are released into the circulation. The absence of glycerol kinase in the adipocyte decreases triacylglycerol resynthesize and supports hepatic gluconeogenesis [40]. After obtaining the overnight fasting blood sample, the serum will be separated. The serum sample will be stored at - 
$70{ }^{\circ} \mathrm{C}$ for further measurement of free glycerol by enzymatic colorimetric method.

6. Glucose homeostasis (fasting blood sugar (FBS), insulin)

7. Inflammatory status (CRP, neutrophil/lymphocyte ratio (NLR))

- NLR is an available measurable marker used to measure systemic inflammation.

8. Dysbiosis status and luminal integrity (endotoxin levels)

- Intestinal gram-negative bacteria are the major source of lipopolysaccharides (LPS), which are referred to as endotoxins. In the case of reduced intestinal barrier integrity due to dysbiosis, luminal endotoxins can enter the circulation [41]. Endotoxin activity assay (EAA) will be used to determine endotoxin levels in whole blood.

\section{Secondary outcomes}

The secondary outcomes are:

1. Enteral feeding tolerance (abdominal examination and GRV measurement)

2. Clinical prognosis (APACHE and SOFA score)

3. Nutritional status (NUTRIC score)

4. Infectious complications incidence

5. Pressure ulcer incidence and grade

6. Ventilator-dependent days

7. Length of ICU stay

8. Length of hospital stay

9. 28-Day mortality

\section{Data analysis}

Data will be analyzed using SPSS for windows version 11.5 and MedCalc Statistical Software version 18.11.3 (MedCalc Software bvba, Ostend, Belgium; https://www. medcalc.org; 2019). Descriptive (frequency, percentage, mean, standard deviation) and inferential analysis (student $t$ test, paired sample $t$ test, repeated measures analysis of variance (ANOVA)) will be performed. Any covariates will be controlled by ANCOVA or binary logistic regression.
All tests will be two-tailed and a $p$ value $<0.05$ will be considered as statistically significant.

\section{Data collection and quality assurance}

Data collection will be supervised by the primary investigator. In addition, $10 \%$ of electronic data will be checked randomly with paper questionnaires and any discrepancies will lead to a $50 \%$ double-checking of electronic data. Any outliers will be checked against patient medical records.

\section{Discussion}

In the intestinal tract, gut microbiota control different immune and endocrine functions [42]. They have a major role in the absorption, storage, and consumption of energy derived from the diet $[14,15]$. Outside the intestine, they also modulate cell metabolism, energy homeostasis, systemic inflammation, appetite and food intake [42, 43]. On the other hand, a few clinical studies, modulating gut microbiota in patients under critical care demonstrated a faster return of gut function and earlier achievement of the nutritional target dose $[16,17]$. Therefore, we expect that our patients in the intervention group will have a better enteric feeding tolerance and also more desirable energy homeostasis.

Animal studies have shown that modulation of gut microbiota by prebiotics, probiotics, or synbiotics can reduce cachexia and muscle mass sparing [22-25]. The underlying mechanisms remain to be identified. Gut microbiota influence amino acid bioavailability and are a source of different metabolites such as conjugated linoleic acid, acetate, and bile acids that modulate muscle metabolism. Gut microbiota are also a source of PAMPs, which activate the transcription factor NF-kB through toll-like receptors (TLRs) and cause muscle wasting. Gut microbiota also modulate production of proinflammatory cytokines, which can induce muscle atrophy [8]. We expect that synbiotic intervention in patients under critical care reduces muscle protein degradation and turnover. As malnutrition and muscle wasting in these patients are associated with negative health outcomes, gut microbiota modulation will improve the clinical prognosis and reduce infectious complications, ventilator dependency, and length of ICU stay.

\section{Trial status}

Recruitment was started on 1 March 2019 and is estimated to be completed by October 2019. Recruitment was ongoing at the time of submission. This is the last protocol version (Number 5, 15 January 2020).

\section{Additional file}

Additional file 1. Standard protocol items: recommendation for interventional trials (SPIRIT) 2013 Checklist: Recommended items to address in a clinical trial protocol and related documents. 


\section{Abbreviations}

3MH: 3-Methy histidine; AIDS: Acquired immune deficiency syndrome; ALT: Alanine aminotransferase; ANCOVA: Analysis of covariance; ANOVA: Analysis of variance; APACHE II: Acute Physiology and Chronic Health Evaluation II; AST: Aspartate aminotransferase; CFU: Colony-forming units; Cl: Chloride; Cr: Creatinine; CRP: C-reactive protein; EAA: Endotoxin activity assay; ELISA: Enzyme-linked immunosorbent assay; EN: Enteral nutrition; ESPEN: European Society of Parenteral and Enteral Nutrition; GCS: Glasgow coma scale; GIT: Gastrointestinal tract; GRV: Gastric residual volume; ICU: Intensive care unit; K: Potassium; LPS: Lipopolysaccharides; Mg: Magnesium; MOF: Multi-organ failure; Na: Sodium; NF-kB: Nuclear factor kappa light chain enhancer of activated B cells; NGT: Nasogastric tube; NLR: Neutrophillymphocyte ratio; NUTRIC: Nutrition risk in critically ill; P: Phosphorus; PAMPs: Pathogen-associated molecular patterns; PreAlb: Prealbumin; SOFA: Sequential Organ Failure Assessment; TG: Triglyceride; TLRs: Toll-like receptors

\section{Acknowledgements}

The support provided by Mashhad University of Medical Sciences (MUMS) to conduct this study is highly acknowledged.

\section{Confidentiality}

Participants' study information will be stored at the security site. All laboratory specimens, data collected, and reports will be identified by a coded ID number.

\section{Dissemination}

We will attempt to release the full study protocol and results as soon as possible, regardless of the magnitude or direction of effect. The anonymized data set and statistical code may be available from the corresponding author on reasonable request.

\section{Authors' contributions}

NS and MS initially conceptualized and designed the study. MS, AS, MN, and RR upgraded the protocol design and contributed to obtaining initial funding. The manuscript was written by NS and reviewed by all members. MKR was responsible for design optimizing and statistical analysis. All authors read and approved the final manuscript.

\section{Funding}

This research will be funded by the vice chancellery for research of Mashhad University of Medical Sciences (MUMS), and all study stages will be undertaken under its supervision.

\section{Availability of data and materials}

The datasets generated and/or analyzed during the current study are not publicly available due to ethical considerations, but may be available from the corresponding author on reasonable request.

\section{Ethics approval and consent to participate}

Ethical approval was obtained from ethical committee of MUMS. The ethical approval code is IR.MUMS.MEDICAL.REC.1397.715. The informed consent will be obtained from all study participants or their legal guardian. Any modification to protocol which may impact on the conduct of the study, will be approved by ethical committee of MUMS prior to implementation.

\section{Consent for publication}

No personal identifying information will be published.

\section{Competing interests}

The authors declare that they have no competing interests.

\section{Author details}

'Department of Nutrition, Medical School, Mashhad University of Medical Sciences, Mashhad, Iran. ${ }^{2}$ Metabolic Syndrome Research Center, Mashhad University of Medical Sciences, Mashhad, Iran. ${ }^{3}$ Department of community medicine, Medical School, Mashhad University of Medical Sciences, Mashhad, Iran. ${ }^{4}$ Department of Anesthesiology, Mashhad University of Medical Sciences, Mashhad, Iran.
Received: 1 October 2019 Accepted: 3 February 2020

Published online: 24 February 2020

\section{References}

1. Sekirov I, Russell SL, Antunes LC, Finlay BB. Gut microbiota in health and disease. Physiol Rev. 2010;90(3):859-904.

2. Gilbert JA, Blaser MJ, Caporaso JG, Jansson JK, Lynch SV, Knight R. Current understanding of the human microbiome. Nat Med. 2018;24(4):392.

3. Young VB. The role of the microbiome in human health and disease: an introduction for clinicians. Bmj. 2017;356:j831.

4. Stefanaki C, Bacopoulou F, Michos A. The impact of probiotics' administration on glycemic control, body composition, gut microbiome, mitochondria, and other hormonal signals in adolescents with prediabetesa randomized, controlled trial study protocol. Contemp Clin Trials Commun. 2018;11:55-62.

5. Feng $Q$, Chen W-D, Wang Y-D. Gut microbiota: an integral moderator in health and disease. Front Microbiol. 2018;9:151.

6. Wischmeyer PE, McDonald D, Knight R. Role of the microbiome, probiotics, and 'dysbiosis therapy' in critical illness. Curr Opin Crit Care. 2016;22(4):347-53.

7. Alverdy JC, Chang EB. The re-emerging role of the intestinal microflora in critical illness and inflammation: why the gut hypothesis of sepsis syndrome will not go away. J Leukoc Biol. 2008;83(3):461-6.

8. Bindels $L B$, Delzenne NM. Muscle wasting: the gut microbiota as a new therapeutic target? Int J Biochem Cell Biol. 2013;45(10):2186-90.

9. Klein GL, Petschow BW, Shaw AL, Weaver E. Gut barrier dysfunction and microbial translocation in cancer cachexia: a new therapeutic target. Curr Opin Support Palliat Care. 2013;7(4):361.

10. Quirk J. Malnutrition in critically ill patients in intensive care units. Br J Nurs. 2000;9(9):537-41.

11. Lew CCH, Yandell R, Fraser RJ, Chua AP, Chong MFF, Miller M. Association between malnutrition and clinical outcomes in the intensive care unit: a systematic review. J Parenter Enter Nutr. 2017;41(5):744-58.

12. Lew C, Wong G, Cheung K, Chua A, Chong M, Miller M. Association between malnutrition and 28-day mortality and intensive care length-ofstay in the critically ill: a prospective cohort study. Nutrients. 2018;10(1):10.

13. Cederholm T, Bosaeus I, Barazzoni R, Bauer J, Van Gossum A, Klek S, et al. Diagnostic criteria for malnutrition-an ESPEN consensus statement. Clin Nutr. 2015;34(3):335-40.

14. Duca F, Lam T. Gut microbiota, nutrient sensing and energy balance. Diabetes Obes Metab. 2014;16(S1):68-76.

15. Blaut M. Gut microbiota and energy balance: role in obesity. Proc Nutr Soc. 2015;74(3):227-34

16. Tuncay P, Arpaci F, Doganay M, Erdem D, Sahna A, Ergun H, et al. Use of standard enteral formula versus enteric formula with prebiotic content in nutrition therapy: a randomized controlled study among neuro-critical care patients. Clin nutr ESPEN. 2018;25:26-36.

17. Malik AA, Rajandram R, Tah PC, Hakumat-Rai V-R, Chin K-F. Microbial cell preparation in enteral feeding in critically ill patients: a randomized, doubleblind, placebo-controlled clinical trial. J Crit Care. 2016;32:182-8.

18. Sanaie S, Ebrahimi-Mameghani M, Hamishehkar H, Mojtahedzadeh M, Mahmoodpoor A. Effect of a multispecies probiotic on inflammatory markers in critically ill patients: a randomized, double-blind, placebocontrolled trial. J Res Med Sci. 2014;19(9):827-33.

19. Manzanares W, Lemieux M, Langlois PL, Wischmeyer PE. Probiotic and synbiotic therapy in critical illness: a systematic review and meta-analysis. Crit Care. 2016;20(1):262.

20. Spiljar M, Merkler D, Trajkovski M. The immune system bridges the gut microbiota with systemic energy homeostasis: focus on TLRs, mucosal barrier, and SCFAs. Front Immunol. 2017;8:1353.

21. Landi F, Camprubi-Robles M, Bear DE, Cederholm T, Malafarina V, Welch AA, et al. Muscle loss: the new malnutrition challenge in clinical practice. Clin Nutr. 2019;8(5):2113-20.

22. Bindels LB, Beck R, Schakman O, Martin JC, De Backer F, Sohet FM, et al. Restoring specific lactobacilli levels decreases inflammation and muscle atrophy markers in an acute leukemia mouse model. PLoS One. 2012;7(6):e37971.

23. Bindels LB, Neyrinck AM, Salazar N, Taminiau B, Druart C, Muccioli GG, et al. Non digestible oligosaccharides modulate the gut microbiota to control the development of leukemia and associated cachexia in mice. PLoS One. 2015; 10(6):e0131009. 
24. Bindels LB, Neyrinck AM, Claus SP, Le Roy Cl, Grangette C, Pot B, et al. Synbiotic approach restores intestinal homeostasis and prolongs survival in leukaemic mice with cachexia. The ISME journal. 2016;10(6):1456-70.

25. Varian BJ, Goureshetti S, Poutahidis T, Lakritz JR, Levkovich T, Kwok C, et al. Beneficial bacteria inhibit cachexia. Oncotarget. 2016;7(11):11803-16.

26. Singer P, Blaser AR, Berger MM, Alhazzani W, Calder PC, Casaer MP, et al. ESPEN guideline on clinical nutrition in the intensive care unit. Clin nutri. 2019;38(1):48-79.

27. Aguilera-Martinez R, Ramis-Ortega E, Carratalá-Munuera C, FernándezMedina JM, Saiz-Vinuesa MD, Barrado-Narvión MJ. Effectiveness of continuous enteral nutrition versus intermittent enteral nutrition in intensive care patients: a systematic review. JBI Database System Rev Implement Rep. 2014;12(1):281-317.

28. Patel JJ, Rosenthal MD, Heyland DK. Intermittent versus continuous feeding in critically ill adults. Curr Opin Clin Nutr Metab Care. 2018:21(2):116-20.

29. Williams NT. Probiotics. Am J Health Syst Pharm. 2010;67(6):449-58.

30. Holzer P. Treatment of opioid-induced gut dysfunction. Expert Opin Investig Drugs. 2007;16(2):181-94

31. Balzan S, de Almeida QC, De Cleva R, Zilberstein B, Cecconello I. Bacterial translocation: overview of mechanisms and clinical impact. J Gastroenterol Hepatol. 2007;22(4):464-71.

32. Kanno T, Matsuki T, Oka M, Utsunomiya H, Inada K, Magari H, et al. Gastric acid reduction leads to an alteration in lower intestinal microflora. Biochem Biophys Res Commun. 2009:381(4):666-70

33. Vitko HA, Troxell JJ. Probiotics in the critical care unit: fad, fact, or fiction? J Emerg Crit Care Med. 2018:2:95-106

34. Berger MM, Reintam-Blaser A, Calder PC, Casaer M, Hiesmayr MJ, Mayer K, et al. Monitoring nutrition in the ICU. Clin Nutr. 2019;38(2):584-93.

35. Mahmoodpoor A, Hamishehkar H, Asghari R, Abri R, Shadvar K, Sanaie S. Effect of a probiotic preparation on ventilator-associated pneumonia in critically ill patients admitted to the intensive care unit: a prospective double-blind randomized controlled trial. Nutr Clin Pract. 2019;34(1):156-62.

36. Pupim LB, Martin CJ, Ikizler TA. Assessment of protein and energy nutritional status. Nutritional Management of Renal Disease. Academic Press; 2013. p. 137-58.

37. Dickerson RN. Nitrogen balance and protein requirements for critically ill older patients. Nutrients. 2016:8(4):226

38. Elia M, Carter A, Bacon S, Winearls C, Smith R. Clinical usefulness of urinary 3-methylhistidine excretion in indicating muscle protein breakdown. Br Med J (Clin Res Ed). 1981;282(6261):351-4.

39. Kochlik B, Gerbracht C, Grune T, Weber D. The influence of dietary habits and meat consumption on plasma 3-methylhistidine-a potential marker for muscle protein turnover. Mol Nutr Food Res. 2018;62(9):1701062.

40. Ahmadian M, Wang Y, Sul HS. Lipolysis in adipocytes. Int J Biochem Cell Biol. 2010;42(5):555-9.

41. Mani V. Understanding intestinal lipopolysaccharide permeability and associated inflammation. PhD [dissertion on the Internet]. Ames, lowa: lowa State University; 2012 [cited 24 Nov 2019]. Available from: https://lib.dr. iastate.edu/cgi/viewcontent.cgi?article=3795\&context=etd;Understanding .

42. Delzenne NM, Cani PD. Interaction between obesity and the gut microbiota: relevance in nutrition. Annu Rev Nutr. 2011;31:15-31.

43. Kairupan TS, Amitani H, Cheng K-C, Runtuwene J, Asakawa A, Inui A. Role of gastrointestinal hormones in feeding behavior and obesity treatment. J Gastroenterol. 2016;51(2):93-103.

\section{Publisher's Note}

Springer Nature remains neutral with regard to jurisdictional claims in published maps and institutional affiliations.

Ready to submit your research? Choose BMC and benefit from:
- fast, convenient online submission
- thorough peer review by experienced researchers in your field
- rapid publication on acceptance
- support for research data, including large and complex data types
- gold Open Access which fosters wider collaboration and increased citations
- maximum visibility for your research: over 100M website views per year
At BMC, research is always in progress.
Learn more biomedcentral.com/submissions

